\begin{tabular}{|c|l|}
\hline Title & Scattering-Based Stochastic Process A ttracting Electrons I nward under A ntiparallel Gradient Magnetic Fields \\
\hline Author(s) & Sugawara, Hirotake \\
\hline Citation & $\begin{array}{l}\text { Journal of the Physical Society of Japan, 82(6), 064501 } \\
\text { https://doi.org/L0.7566/PSJ.82.064501 }\end{array}$ \\
\hline Issue Date & 2013-06 \\
\hline Doc URL & http://hdl.handle.net/2115/53413 \\
\hline Rights & ○ 2013 The Physical Society of Japan \\
\hline Type & article (author version) \\
\hline File Information & Sugawara 2013-JPSJ-HUSCAP.pdf \\
\hline
\end{tabular}

Instructions for use 


\title{
Scattering-Based Stochastic Process Attracting Electrons Inward under Antiparallel Gradient Magnetic Fields*
}

\author{
Hirotake SugAWARA $^{\dagger}$ \\ Graduate School of Information Science and Technology, Hokkaido University, North \\ 14 West 9, Sapporo 060-0814, Japan
}

Electron gyration and scattering in a gas under antiparallel gradient magnetic fields (APGMFs) and rf electric field were simulated using a Monte Carlo method to identify the factors of the electron confinement effect of the APGMFs. The inward shift of the gyrocenter at electron scattering worked as an additional factor to electron meandering, which has been regarded as the primary factor of the electron confinement. This scattering-based inward electron attraction occurs by a simple principle that the gyroradius and residence time of electrons become greater under weaker magnetic fields in the inner region of the APGMFs.

KEYWORDS: antiparallel gradient magnetic fields, electron confinement, electron gyration, magnetized plasma, Monte Carlo simulation, scattering

\section{Introduction}

Antiparallel gradient magnetic fields (APGMFs) are a model field configuration for the analysis of fundamental electron behavior in a magnetic neutral loop discharge (NLD) plasma. The NLD plasma is a low-pressure inductively coupled magnetized plasma used for dry etching. ${ }^{1,2)}$ The NLD plasma is generated along a ring of zero magnetic field named the neutral loop (NL), and the magnetic field around the NL consists of two pairs of APGMFs.

The APGMFs are known to have a function of electron confinement, under which the electron distribution lateral to the NL has a certain breadth and the electron conduction path is formed along the NL. ${ }^{3,4}$ ) This electron conduction is regarded as a loop plasma current macroscopically ${ }^{5)}$ and plays a key role in the sustainment of the NLD plasma as the recipient of the electric power supplied through inductive coupling with an $\mathrm{rf}$

\footnotetext{
${ }^{*}$ Published source: Journal of the Physical Society of Japan 82(6) 064501 (6 pages) (2013) Jun/2013, DOI: $10.7566 /$ JPSJ.82.064501

${ }^{\dagger}$ E-mail address: sugawara@ist.hokudai.ac.jp
} 


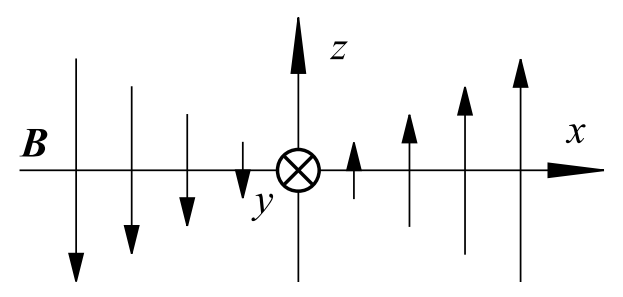

Fig. 1. Antiparallel gradient magnetic fields. $\boldsymbol{B}=\left(B_{x}, B_{y}, B_{z}\right)=(0,0, \beta x)$, where $\beta=\mathrm{d} B_{z} / \mathrm{d} x>0$ is a constant. A uniform rf electric field is applied in the $y$-direction.

antenna. The chaotic electron meandering ${ }^{6-8)}$ and resulting collisionless heating ${ }^{9)}$ in the APGMFs have been investigated as fundamental processes. However, details of the confinement mechanism have been left unexplained.

In this paper, the factors of the electron confinement by the APGMFs are discussed. A stochastic inward shift of the electron gyration range induced by scattering due to electron-molecule collisions is recognized as one of them. Simulation data supporting this conclusion are presented.

\section{Field Model}

After preceding investigations, ${ }^{3,4,6-9)}$ the APGMFs were defined in a boundary-free space as

$$
\boldsymbol{B}=\left(B_{x}, B_{y}, B_{z}\right)=(0,0, \beta x)
$$

where $\beta=\mathrm{d} B_{z} / \mathrm{d} x$ was assumed to be a positive constant (see Fig. 1). The linearity of the $\boldsymbol{B}$ field near the positions of $|\boldsymbol{B}|=0$ is seen in measurement ${ }^{10)}$ and calculation results. ${ }^{11)}$ This $\boldsymbol{B}$ field can be formed by two parallel sheet currents. ${ }^{3,7)}$

An rf electric field was applied uniformly in the $y$-direction as

$$
\boldsymbol{E}=\left(E_{x}, E_{y}, E_{z}\right)=(0, E \sin (2 \pi f t+\phi), 0),
$$

where $f=13.56 \mathrm{MHz}$. The effect of space charge field was neglected as in the electron swarm theory to clearly identify the essential characteristics of the electron motion in the APGMFs.

The electrons distributed near the $y z$-plane and electron conduction occurred in the $y$-direction.

\section{Fundamental Electron Behavior}

First, a brief overview of the electron behavior in the $\mathrm{APGMFs}^{3,4)}$ is presented. After that, an example of single electron motion in the APGMFs and the lateral electron 
distribution are shown to point out two key properties indicating the presence of the process attracting electrons inward through scattering.

\subsection{Overview}

Electrons meander in the weak magnetic field near $x=0$ and gyrate in the strong magnetic field distant from $x=0$.

When $E_{y}<0$, the electrons are accelerated forward, i.e., toward the $+y$-direction. The Lorentz force makes the electrons flying forward turn inward, which induces the meandering. The meandering electrons can drift forward and gain energy. If the period of $E_{y}<0$ is sufficiently long, even gyrating electrons approach $x=0$ and eventually transfer from the gyration region to the meandering region because the $\boldsymbol{E} \times \boldsymbol{B}$ drift is inward.

When $E_{y}>0$, oppositely, the electrons are accelerated backward, i.e., toward the $-y$-direction. They turn outward, start gyration, and deviate from $x=0$ by the outward $\boldsymbol{E} \times \boldsymbol{B}$ drift. They hardly drift backward.

Thus, the APGMFs are conductive for the forward electron flow and resistive for the backward one. Under the $\operatorname{rf} \boldsymbol{E}$ field, the conductive and resistive modes alternate every half an rf period.

\subsection{Single electron motion}

Figure 2 shows the meandering and gyration in the APGMFs. The $\boldsymbol{B}$ and $\boldsymbol{E}$ fields were set as $\beta=0.5 \mathrm{mT} \mathrm{cm}^{-1}, E=-10.0 \mathrm{~V} \mathrm{~cm}^{-1}$, and $\phi=0$. The electron trajectory was calculated using the Runge-Kutta method with a time step $\Delta t=3.69 \mathrm{ps}$, which was $1 / 20000$ of the $\operatorname{rf}$ period $T=1 / f=73.7 \mathrm{~ns}$ and shorter than $1 / 1000$ of the gyration periods dealt with in this simulation. The electron-molecule collisions were simulated using a Monte Carlo method with a time-saving judgment scheme for collisional events. ${ }^{12)}$ The gas was assumed to be $\mathrm{CF}_{4}$ at a molecule number density of $N=1.77 \times 10^{13} \mathrm{~cm}^{-3}(0.067 \mathrm{~Pa}$ at $273 \mathrm{~K})$. The electron collision cross sections of $\mathrm{CF}_{4}$ were obtained from ref. 13. The electron was released from the origin with a velocity corresponding to $5.4 \mathrm{eV}$, which was chosen randomly from a Maxwellian distribution with a mean energy of $10 \mathrm{eV}$. The broken lines represent the positions of the rf-resonant magnetic field strength for reference; the electron cyclotron resonance occurs at $B_{\mathrm{ECR}}=2 \pi m f / e=0.484 \mathrm{mT}$ at $f=13.56 \mathrm{MHz}$, where $e$ and $m$ are the electronic charge and mass, respectively. 


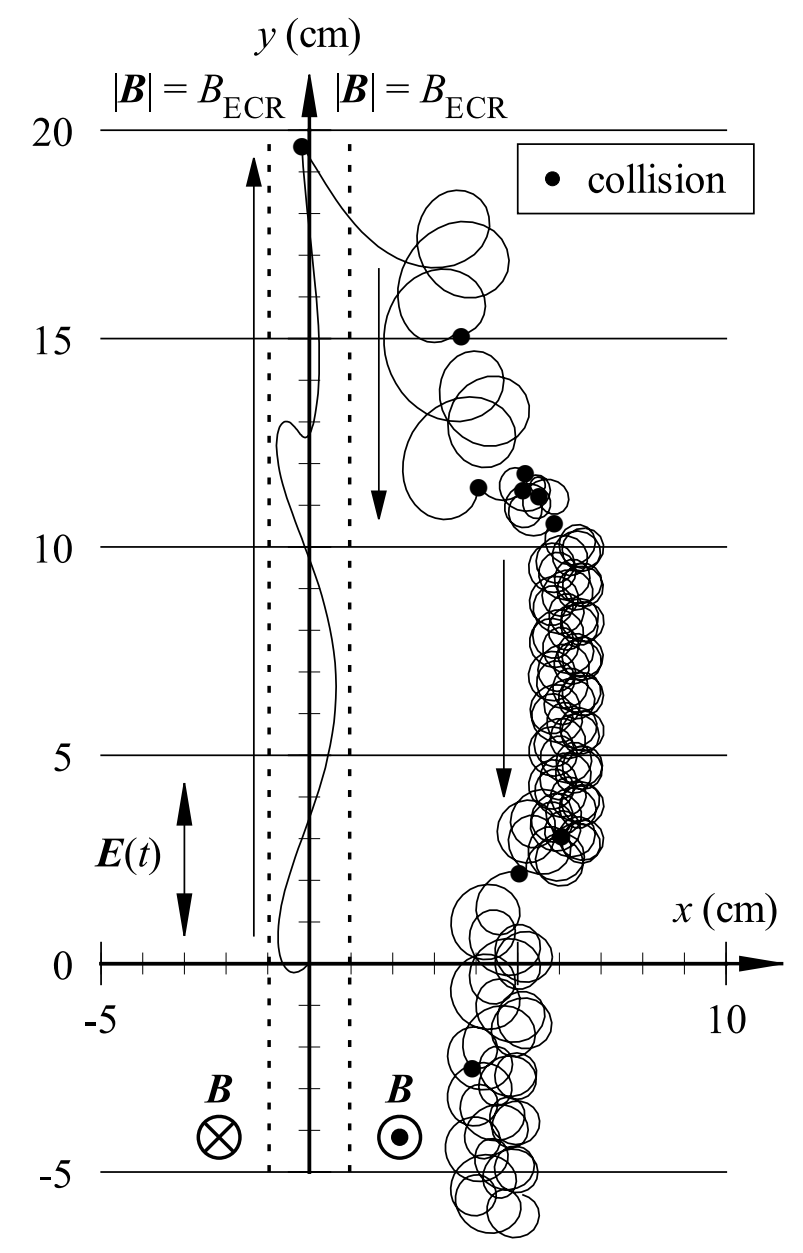

Fig. 2. Example of electron locus.

The electron first meandered forward. It deviated from $x=0$ after the collision near $y=20 \mathrm{~cm}$ and started gyration. The backward drift was induced by a variation in the gyroradius $r=m|\boldsymbol{v}| /(e|\boldsymbol{B}|)$ depending on the $x$-position via $|\boldsymbol{B}|=|\beta x|$, where $\boldsymbol{v}$ is the electron velocity projected to the $x y$-plane. This drift is in principle the same as the grad- $B$ drift at $\boldsymbol{E}=0$. The rf $\boldsymbol{E}$ field induced the libration of the gyrocenter in the $\pm x$-directions via the $\boldsymbol{E} \times \boldsymbol{B}$ drift, e.g., in a flight from $y=10$ to $4 \mathrm{~cm}$. However, the $x$ range was unchanged during a free gyration between two successive collisions as reported previously. ${ }^{14)}$ The electron transferred from one gyration orbit to another through scattering. The $x$ range of gyration varied at that time.

Property 1: The $x$ range of electron gyration is unchanged during a collisionless gyration if the range is sufficiently distant from $x=0$. 


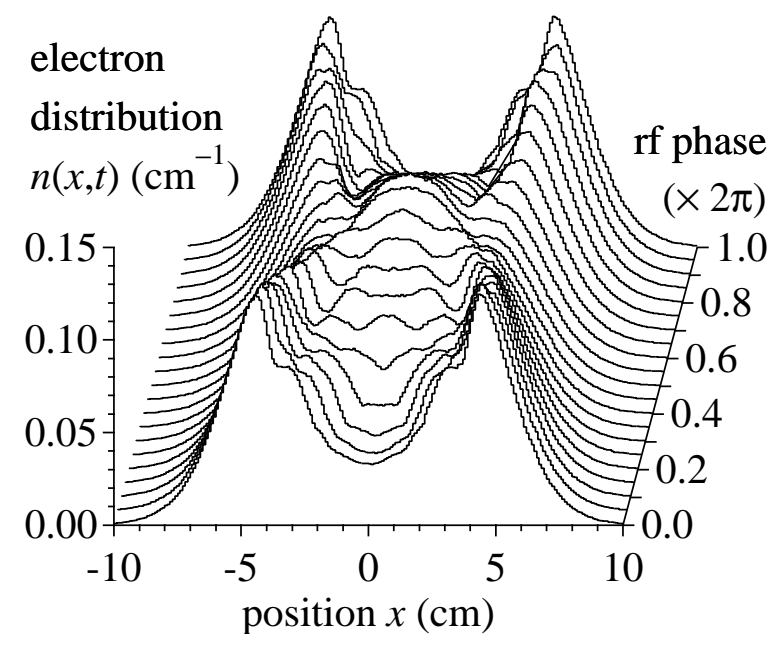

Fig. 3. Phase-resolved lateral electron distribution $n(x, t)$ for an $\mathrm{rf}$ period.

\subsection{Lateral electron distribution}

Figure 3 shows the phase-resolved response of the lateral electron distribution $n(x, t)$ for an rf period in a periodical steady state under the same condition as in Fig. 2. More than $10^{6}$ electrons were sampled after $t=50 T$ to obtain $n(x, t)$. This distribution, which has been normalized to satisfy $\int_{-\infty}^{\infty} n(x, t) \mathrm{d} x=1$, represents the probability density of the presence of electron expected from the single electron motion. The electron number density profile in the $x$-direction to be measured experimentally in number per unit volume would be given as $n_{y z} n(x, t)$, where $n_{y z}$ is an arbitrary scaling factor in number per unit area representing the areal number density of electrons projected onto the $y z$-plane.

The two peaks moved inward during the rf phase $0-\pi$ and outward during $\pi-2 \pi$ in the same way as the libration of the gyrocenter. The electrons remained around $x=0$ with a certain breadth of $n(x, t)$. This breadth no longer expanded after reaching the periodical steady state.

Property 2: The breadth of $n(x, t)$ is unchanged even with the gradient of the electron number density, which may induce outward electron diffusion.

\section{Inward Electron Attraction}

\subsection{Cause of inward electron attraction}

Property 2 implies that the APGMFs must attract the electrons inward to cancel the outward electron diffusion. The electron meandering near $x=0$ is one of such functions. However, the mechanism attracting the electrons gyrating far from $x=0$ inward has 


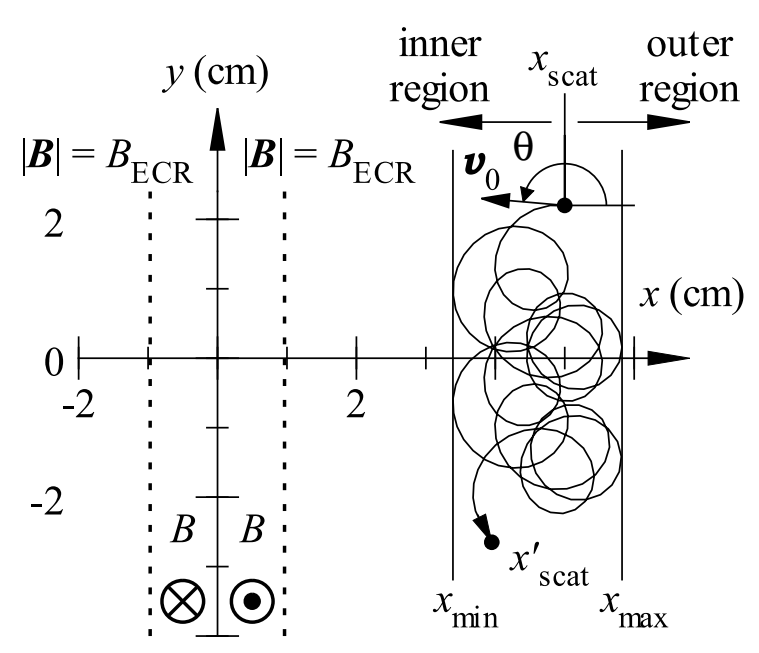

Fig. 4. Range of electron motion in the antiparallel gradient magnetic fields. $x_{\text {scat }}$ and $x_{\text {scat }}^{\prime}$ represent the $x$-positions of two successive collisions, i.e., the start and end of an electron flight, respectively.

been left unexplained. Let us call this mechanism the inward electron attraction (IEA) hereafter.

The IEA by the APGMFs is different from the magnetic mirror effect known as a representative confinement effect of magnetic field. The IEA occurs in the direction perpendicular to the magnetic field, while a magnetic mirror reflects electrons in the direction along the magnetic field without electron-molecule collisions. Property 1 indicates that the IEA would not occur upon stably gyrating electrons if no scattering. The IEA is a stochastic process due to collisions.

\subsection{Definitions of quantities}

Let us define the quantities to describe the range of electron motion as shown in Fig. 4. Consider that an electron is scattered at $x=x_{\text {scat }}>0$ at phase $0 \leq \phi<2 \pi$ of the $\boldsymbol{E}$ field. The following discussion does not lose its generality by this limit for the symmetry of the $\boldsymbol{B}$ field. The electron starts a flight from $x_{\text {scat }} . \theta$ is the angle between the initial electron velocity $\boldsymbol{v}_{0}$ projected to the $x y$-plane and the $x$-axis. The regions of $x<x_{\text {scat }}$ and $x>x_{\text {scat }}$ are named the inner and outer regions, respectively. $x_{\min }$ and $x_{\max }$ represent the $x$ range of the electron flight. The electron flight is terminated at $x=x_{\text {scat }}^{\prime}$ by the succeeding scattering. This $x_{\text {scat }}^{\prime}$ is the start of the succeeding flight.

The average electron position $x_{\text {ave }}$ during a flight time $\tau$ is defined with the weight 
of residence time $\mathrm{d} t$ at $x$ as

$$
x_{\mathrm{ave}}=\frac{1}{\tau} \int_{0}^{\tau} x(t) \mathrm{d} t .
$$

When the electron gyration orbit is stable under a collisionless condition, $x_{\text {ave }}$ approximates the average position of the gyrocenter in the limit $\tau \rightarrow \infty$.

\subsection{Outline of theory}

The IEA for gyrating electrons is explained in the following steps. ${ }^{15)}$ Here, $\left\langle x_{\text {ave }}\right\rangle$ and $\left\langle x_{\text {scat }}^{\prime}\right\rangle$ denote the averages of $x_{\text {ave }}$ and $x_{\text {scat }}^{\prime}$, respectively, among a group of electrons starting from a common $x_{\text {scat }}$.

(1) The gyroradius is larger in the inner region than in the outer region. This is the given circumstance.

(2) Then, the residence time in the inner region is also longer than that in the outer region on average, i.e., $\left\langle x_{\text {ave }}\right\rangle<x_{\text {scat }}$.

(3) Thus, scatterings terminating the gyrations occur more in the inner region than in the outer region, i.e., $\left\langle x_{\text {scat }}^{\prime}\right\rangle<x_{\text {scat }}$.

(4) Scattering induces the displacement of the range of electron gyration. This displacement can be both inward and outward. However, under the circumstance of step (3), the inward displacement exceeds the outward one among the electrons starting from a common $x_{\text {scat }}$. This induces the IEA.

(5) The outward displacement regarded as the diffusion balances with the inward displacement from the outer region. This is an interpretation of property 2 .

Simulation data for quantifying the aspects in steps (2)-(5) are presented in the following sections. Because the electron motion in the APGMFs is described by a nonlinear differential equation called Duffing's equation ${ }^{7,8)}$ and thus it seemed difficult to analytically confirm the aspects, a numerical approach was adopted in this investigation.

\subsection{Average position of gyrating electron}

Figure 5 shows the distributions of $x_{\min }$ and $x_{\max }$ for about 20000 electrons released from $x_{\text {scat }}=0-10 \mathrm{~cm}$. Their $\boldsymbol{v}_{0}$ and $\phi$ were chosen randomly from a Maxwellian distribution with a mean energy of $10 \mathrm{eV}$ assuming isotropic scattering and from $0-2 \pi$, respectively. The electrons were traced for $100 T$ with $\Delta t=T / 100000$ under a collisionless condition to approximate $\tau \rightarrow \infty$. The $\boldsymbol{B}$ and $\boldsymbol{E}$ fields were the same as those considered in the preceding simulation. 


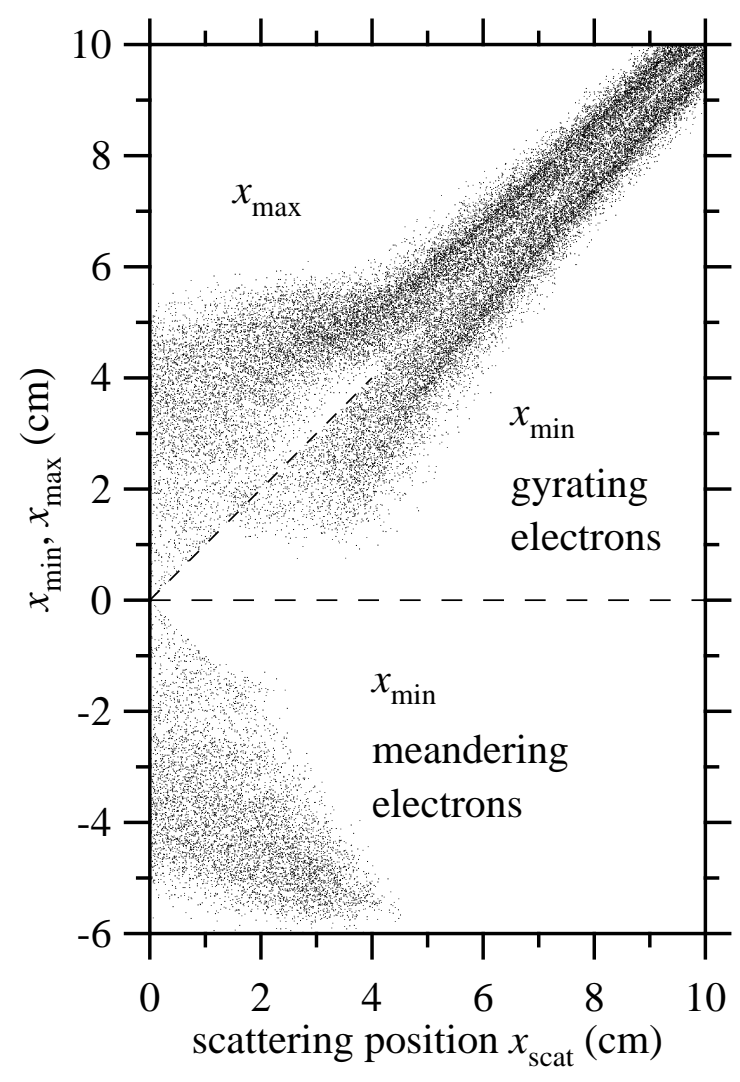

Fig. 5. Distribution of the lower and upper limits of electron motion range, $x_{\min }$ and $x_{\max }$, respectively, for electrons starting from $x=x_{\text {scat }}>0 . x_{\min }>0$ represents gyration and $x_{\min }<0$ represents meandering across $x=0$.

Each $x_{\min }$ has its counterpart $x_{\max }$ at the same $x_{\text {scat }}$. The gyrating electrons are represented by $x_{\min }>0$ in a region of greater $x_{\text {scat }}$ values, and the meandering ones are represented by $x_{\text {min }}<0$ in a region of lesser $x_{\text {scat }}$ values. The meandering electrons seem to distribute between the two peaks of $n(x, t)$ in Fig. 3. However, there is no critical boundary of $x_{\text {scat }}$ separating the gyrating and meandering electrons, because the meandering region is reachable from any $x_{\text {scat }}$ as long as the required $\boldsymbol{v}_{0}$ is given to an electron to make $r \propto|\boldsymbol{v}|$ sufficiently long.

Figure 6 shows the distribution of $x_{\text {ave }}$ of the same electrons as presented in Fig. 5. The $x_{\text {ave }}$ values of the gyrating electrons distribute along the line $x_{\text {ave }}=x_{\text {scat }}$, but have gaps at specific $x$-positions where $|\boldsymbol{B}|$ is a multiple of $B_{\mathrm{ECR}} \cdot x_{\mathrm{ave}}$ cannot locate at such $x$-positions stably perhaps for a type of temporary resonance changing $r, x_{\min }$, and $x_{\max }$ through the electron energy gain or loss. On the other hand, the $x_{\text {ave }}$ values of the electrons that reached the meandering region were effectively zero, because they moved both in $x>0$ and $x<0$ across $x=0$. 


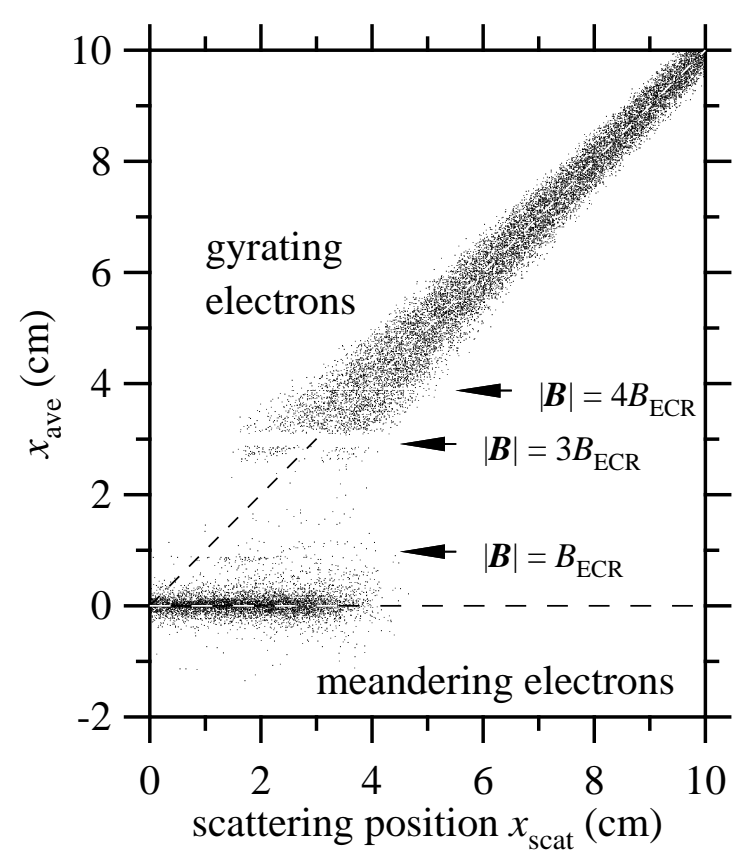

Fig. 6. Distribution of the average position $x_{\text {ave }}$ for electrons starting from $x=x_{\text {scat }}>0$. Those distributing along $x_{\text {ave }}=x_{\text {scat }}$ are of the gyrating electrons.

\subsection{Average of average positions}

Let us see the property $\left\langle x_{\text {ave }}\right\rangle<x_{\text {scat }}$ in step (2) of the outline. Figure 6 shows that both $x_{\text {ave }}>x_{\text {scat }}$ and $x_{\text {ave }}<x_{\text {scat }}$ can occur depending on the initial condition. The parameters for determining the gyration orbit are $\beta, E, x_{\text {scat }}, v_{0}=\left|\boldsymbol{v}_{0}\right|, \theta$, and $\phi$. Since the parameters are too many to examine all possible combinations, the dependence of $x_{\text {ave }}$ on the stochastic parameters $\theta$ and $\phi$ was investigated. To evaluate $\left\langle x_{\text {ave }}\right\rangle$ with an acceptable precision and to show a qualitative change in the electron behavior, $x_{\text {ave was }}$ calculated by varying $\theta$ and $\phi$ uniformly at some $x_{\text {scat }}$ values with fixed $\beta, E$, and $v_{0}$.

Figure 7 shows the variation in $x_{\text {ave }}$ at $\beta=0.5 \mathrm{mT} \mathrm{cm}^{-1}, E=-10.0 \mathrm{~V} \mathrm{~cm}^{-1}$, $\frac{1}{2} m v_{0}^{2}=1 \mathrm{eV}$, and $x_{\text {scat }}=2,4$, and $6 \mathrm{~cm} . \theta$ and $\phi$ were varied from 0 to $2 \pi$ in uniform steps of $\pi / 60$. The electrons were traced for $100 T$ with $\Delta t=T / 120000$ under the collisionless condition.

The distribution range of $x_{\text {ave }}$ became wider with decreasing $x_{\text {scat }}$, which represents the extension of $r$ with decreasing $|\boldsymbol{B}|$. The gaps of $x_{\text {ave }}$ observed in Fig. 6 appeared again. Such a disorder was recognized at least up to $|\boldsymbol{B}|=5 B_{\mathrm{ECR}}$ in an additional finer calculation performed for $x_{\text {scat }}=5 \mathrm{~cm}$ with $\theta$ and $\phi$ values in steps of $\pi / 180$.

Some of the electrons starting from $x_{\text {scat }}=2$ and $4 \mathrm{~cm}$ transferred to meandering. It is considered that this transfer has conventionally been regarded as the confinement 


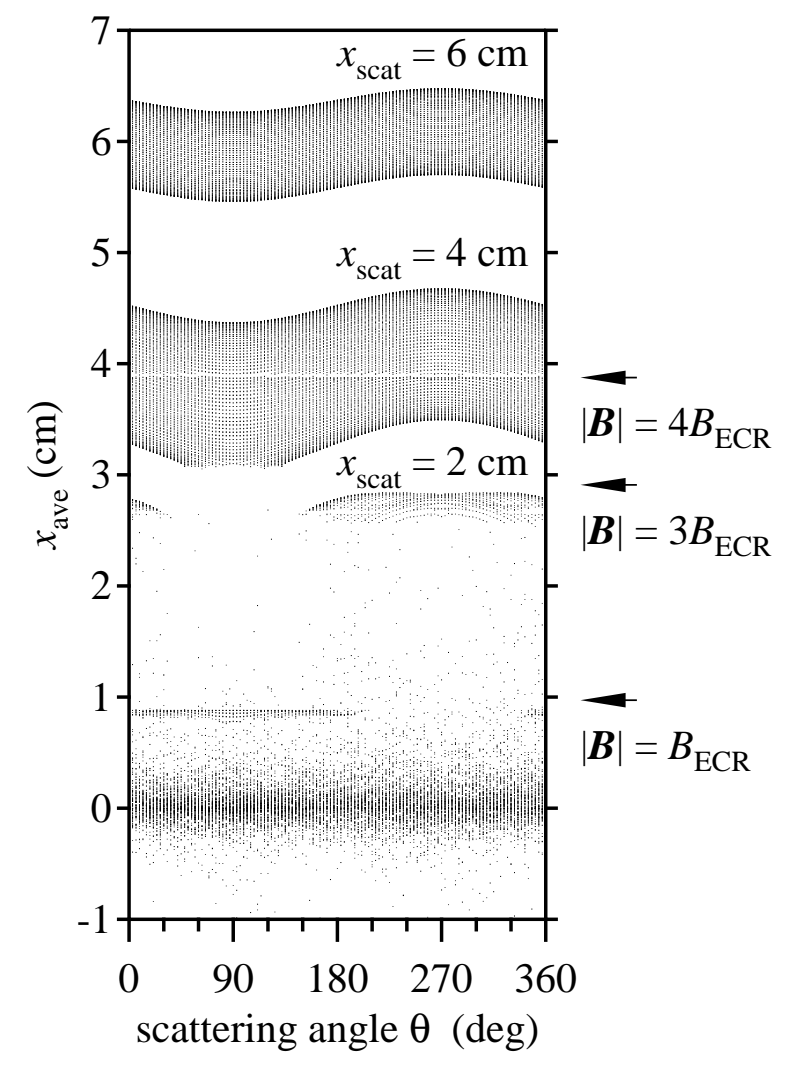

Fig. 7. Average position $x_{\text {ave }}$ after scattering at $x_{\text {scat }}=2,4$, and $6 \mathrm{~cm}$ as a function of the initial direction $\theta$ and initial phase $\phi$ of the applied rf electric field. $\beta=0.5 \mathrm{mT} \mathrm{cm}^{-1}$ and $\frac{1}{2} m v_{0}^{2}=1 \mathrm{eV}$. $0 \leq \theta<2 \pi$ and $0 \leq \phi<2 \pi$ in steps of $\pi / 60$.

effect of the APGMFs together with the meandering itself. The IEA is distinguished from them because it happens even to the gyrating electrons that do not reach the meandering region near $x=0$. The ordered results of $x_{\text {ave }}$ at $x_{\text {scat }}=6 \mathrm{~cm}$ represent such stable orbits that the electrons would not approach the meandering region. For such electrons, Fig. 8 shows $\left\langle x_{\text {ave }}\right\rangle<x_{\text {scat }}$ with small but recognizable position-dependent differences. This result confirms step (2) of the outline. The difference between $\left\langle x_{\text {ave }}\right\rangle$ and $x_{\text {scat }}$ increased with the initial energy for the extension of $r$.

Another finding is that this difference $\left\langle x_{\text {ave }}\right\rangle-x_{\text {scat }}$ obeys the similarity law indicating that the breadth of $n(x, t)$ is proportional to $\left.1 / \sqrt{\beta}{ }^{4}\right)$ Figure 9 shows that the values of $\left(\left\langle x_{\text {ave }}\right\rangle-x_{\text {scat }}\right) \sqrt{\beta}$ calculated at different $\beta$ values show a common curve when they are plotted versus $x_{\text {scat }} \sqrt{\beta}$; i.e., $\left\langle x_{\text {ave }}\right\rangle-x_{\text {scat }} \propto 1 / \sqrt{\beta}$ at fixed $x_{\text {scat }} \sqrt{\beta}$. Their agreement is fine at greater $x_{\text {scat }} \sqrt{\beta}$ values corresponding to the distribution tail of $n(x, t)$, where the temporal variation in $n(x, t)$ is small. No theoretical explanation for this similarity law has been given yet. 


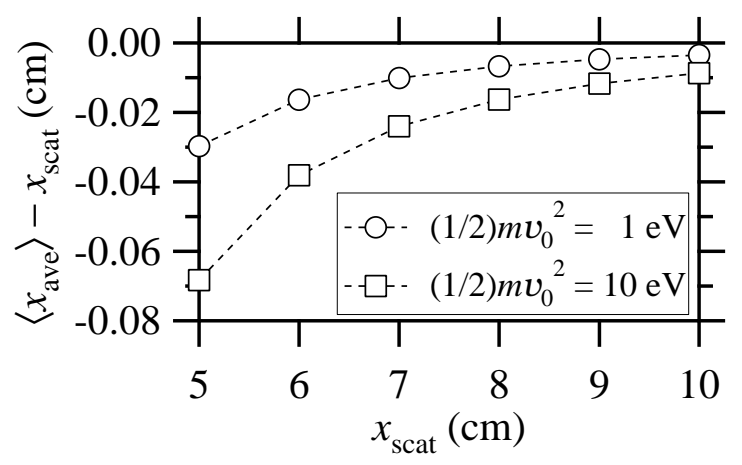

Fig. 8. Average gyrocenter position $\left\langle x_{\text {ave }}\right\rangle$ relative to scattering position $x_{\text {scat }}$.

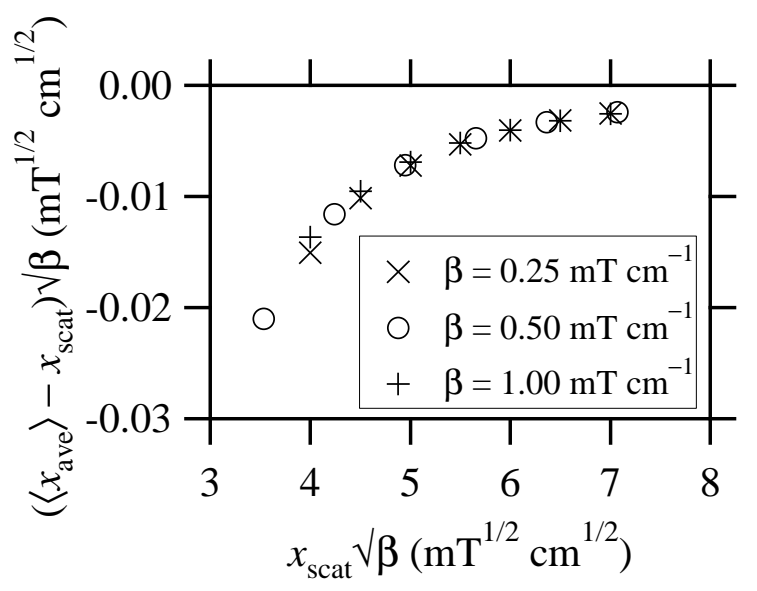

Fig. 9. Normalized relative average gyrocenter position $\left(\left\langle x_{\text {ave }}\right\rangle-x_{\text {scat }}\right) \sqrt{\beta}$ versus normalized scattering position $x_{\text {scat }} \sqrt{\beta} \cdot \frac{1}{2} m v_{0}^{2}=1 \mathrm{eV}$.

\subsection{Position of scattering}

Step (3) of the outline is a convincing inference from step (2). Because the opportunity of electron-molecule collisions during a flight time $\tau$ is more or less proportional to $\tau$ as $\int_{0}^{\tau} N q(v(t)) v(t) \mathrm{d} t \approx \nu \tau, x_{\text {scat }}^{\prime}$ tends to be in the inner region of longer residence time than in the outer region. Here, $q$ is the total electron collision cross section of the ambient gas molecule as a function of the electron speed $v$, and $\nu$ is the collision frequency.

The property $\left\langle x_{\text {scat }}^{\prime}\right\rangle<x_{\text {scat }}$ in step (3) was confirmed under the condition adopted to obtain Fig. 3. Figure 10 shows the $x_{\text {scat }}^{\prime}$ distribution of electrons that started their gyration from $x_{\text {scat }}=1-10 \mathrm{~cm}$. Those electrons that transferred from gyration to meandering were excluded from sampling. The average displacement of the scattering position $\left\langle x_{\text {scat }}^{\prime}\right\rangle-x_{\text {scat }}$ is shown together with a common horizontal scale. 


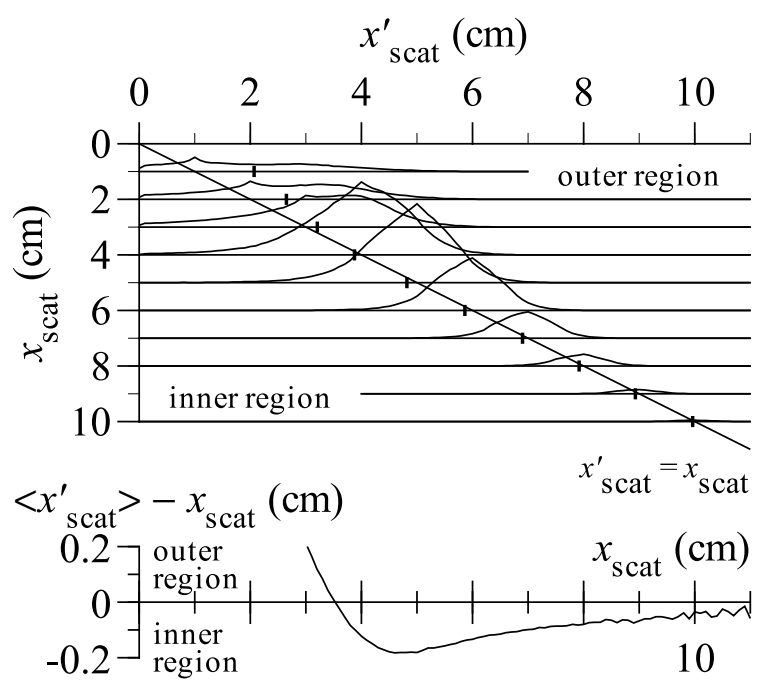

Fig. 10. Distribution of scattering position $x_{\text {scat }}^{\prime}$ of electrons starting their gyration from $x_{\text {scat }}$ (those satisfying $x_{\min }>0$ only). The ticks on the horizontal baselines represent $\left\langle x_{\mathrm{scat}}^{\prime}\right\rangle$. Average displacement of scattering position $\left\langle x_{\text {scat }}^{\prime}\right\rangle-x_{\text {scat }}$ is shown together.

$x_{\text {scat }}^{\prime}$ distributes both in the inner and outer regions. However, $\left\langle x_{\text {scat }}^{\prime}\right\rangle\left\langle x_{\text {scat }}\right.$ for $x_{\text {scat }} \geq 4 \mathrm{~cm}$. This scattering position displacement induced the IEA.

The IEA intensity would be correlated with $\left\langle x_{\text {scat }}^{\prime}\right\rangle-x_{\text {scat }}$ as a function of $x_{\text {scat }}$. An interesting result is that the outer limit of the range in which the peak of $n(x, t)$ moved, around $x=4.5 \mathrm{~cm}$, is close to the position of the minimum $\left\langle x_{\text {scat }}^{\prime}\right\rangle-x_{\text {scat }}$, at which the IEA is considered to be the most intense. The quantification of the IEA in balance with the outward electron diffusion would enable us to derive the factor determining the breadth of $n(x, t)$ in future investigation.

\section{Conclusion}

The mechanism of the IEA induced by electron scattering was recognized as a factor of the electron confinement effect of the APGMFs. In a group of electrons that started gyration from a common position $x_{\text {scat }}$ by isotropic scattering, the time-averaged positions $\left\langle x_{\text {ave }}\right\rangle$ were on average in the inner region relative to $x_{\text {scat }}$. Their difference $\left\langle x_{\text {ave }}\right\rangle-x_{\text {scat }}$ was proportional to $1 / \sqrt{\beta}$ when plotted versus the normalized scattering position $x_{\text {scat }} \sqrt{\beta}$, where $\beta$ is the gradient of the strength of magnetic field. The succeeding scattering tended to occur in the inner region. As a result, the electron gyration range shifted inward on average. This effect balances with the outward electron diffusion owing to the gradient of the electron number density in the lateral electron distribution 
decaying outward. The IEA is a stochastic process, and it occurs even in a region distant from $x=0$. The IEA is distinguished by these features from the conventionally known meandering-based electron confinement effect of the APGMFs.

Some new fundamental features of the electron gyration in the APGMFs were pointed out. The gyrocenter showed libration under the acceleration by the rf electric field. However, in a long-term view, the range of electron gyration was unchanged during a collisionless flight between two successive scatterings when the electron was sufficiently distant from $x=0$. The average position of a gyrating electron, which was regarded as the average gyrocenter, did not stably locate at specific positions at which the strength of magnetic field was a multiple of that of the rf-resonant magnetic field perhaps for the energy gain or loss under a temporary resonance.

The electron behavior in the APGMFs, described by a nonlinear motion equation, was not easily predictable by analytical approaches. Thus, a numerical approach was adopted in the present investigation. Although the examined conditions were only limited combinations of the parameters to determine the electron gyration orbit, the results supported the explanation for the IEA as a stochastic process. The new findings and concept presented in this paper will contribute to further understanding of the electron behavior in the APGMFs.

\section{Acknowledgments}

This work was supported by Grant-in-Aid No. 22540500 from Japan Society for the Promotion of Science and by ULVAC Inc. 


\section{References}

1) T. Uchida: Jpn. J. Appl. Phys. 33 (1994) L43.

2) T. Uchida and S. Hamaguchi: J. Phys. D 41 (2008) 083001.

3) H. Sugawara and Y. Sakai: J. Phys. D 41 (2008) 135208.

4) H. Sugawara, T. Osaga, and H. Yamamoto: Plasma Sources Sci. Technol. 20 (2011) 055002.

5) H. Tsuboi and S. Ogata: Jpn. J. Appl. Phys. 46 (2007) 7475.

6) Z. Yoshida and T. Uchida: Jpn. J. Appl. Phys. 34 (1995) 4213.

7) T. Uchida: J. Vac. Sci. Technol. A 16 (1998) 1529.

8) Z. Yoshida, H. Asakura, H. Kakuno, J. Morikawa, K. Takenuma, S. Takizawa, and T. Uchida: Phys. Rev. Lett. 81 (1998) 2458.

9) H. Asakura, K. Takemura, Z. Yoshida, and T. Uchida: Jpn. J. Appl. Phys. 36 (1997) 4493.

10) T. Sakoda, T. Miyao, K. Uchino, and K. Muraoka: Jpn. J. Appl. Phys. 36 (1997) 6981.

11) T. Gans, D. L. Crintea, D. O'Connell, and U. Czarnetzki: J. Phys. D 40 (2007) 4508.

12) H. Sugawara, N. Mori, Y. Sakai, and Y. Suda: J. Comp. Phys. 223 (2007) 298.

13) M. Kurihara, Z. Lj. Petrović, and T. Makabe: J. Phys. D 33 (2000) 2146.

14) H. Yamamoto, H. Sugawara, and A. Murayama: Proc. Joint Conf. 46th Japan Soc. Appl. Phys. Hokkaido Chapter and 7th Opt. Soc. Japan Hokkaido Region, 2011, C-6 [in Japanese].

15) H. Sugawara: Proc. 21st Europhysics Conf. Atomic and Molecular Physics of Ionized Gases, Viana do Castelo, Portugal, 2012, P1.2.5. 